\title{
Study of Near Consensus Complex Social Networks Using Eigen Theory
}

\author{
Bingo Wing-Kuen Ling \\ School of Engineering, \\ University of Lincoln, \\ Lincoln, LN6 7TS, U.K. \\ wling@lincoln.ac.uk
}

\author{
Paul Stewart \\ School of Engineering, \\ University of Lincoln, \\ Lincoln, LN6 7TS, U.K. \\ pstewart@lincoln.ac.uk
}

\author{
Kok-Lay Teo \\ Dept. of Maths. \& Stat., \\ Curtin U. of Technology, \\ Perth, WA6002, Australia. \\ K.L.Teo@curtin.edu.au
}

\author{
Chi K. Tse \\ Dept. of EIE, \\ Hong Kong Poly. U., \\ Hung Hom, H.K., China. \\ cktse@ieee.org
}

\begin{abstract}
This paper extends the definition of an exact consensus complex social network to that of a near consensus complex social network. A near consensus complex social network is a social network with nontrivial topological features and steady state values of the decision certitudes of the majority of the nodes being either higher or lower than a threshold value. By using eigen theories, the relationships among the vectors representing the steady state values of the decision certitudes of the nodes, the influence weight matrix and the set of vectors representing the initial state values of the decision certitudes of the nodes that satisfies a given near consensus specification are characterized.
\end{abstract}

\section{INTRODUCTION}

A complex network is a network with nontrivial topological features. Complex networks appear in various contexts in our daily life. Over the last decade, complex networks have drawn the attention of many researchers from different fields of physical and social sciences, such as mathematics, computer science, engineering, biology, economics and social sciences. A social network is a network made of individuals, called nodes, which interact with each other. As many social networks have nontrivial topological features, social networks are actually complex networks.

In the past, complex social networks were investigated using lattice and graph theory [1]. Existing studies assume that the complex social networks satisfy an exact consensus property (i.e., the steady state values of the decision certitudes of the nodes are all equal to either 1 or -1 [2]). Because of this strong property, the complex social networks have to contain spanning trees [4], [5]. In reality, the steady state values of the decision certitudes of the majority of the nodes are either higher or lower than a threshold value [3]. Some complex social networks do not contain the spanning trees [4], [5]. Thus, it is useful to extend the definition of the exact consensus property to that of a near consensus property so that the conditions on containing the spanning trees are relaxed. In this paper, the near consensus property is referred to the property that the steady state values of the decision certitudes of the majority of the nodes are either higher or lower than a threshold value. Deriving the near consensus property is a key to the analysis of practical social networks [3]. However, by extending the definition to the near consensus property, the relationships among the vectors representing the steady state values of the decision certitudes of the nodes, the influence weight matrix and the set of vectors representing the initial state values of the decision certitudes of the nodes that satisfies a given near consensus specification are unknown. The aim of this paper is to address the above issues.

Note that this investigation is challenging as the set of vectors representing the steady state values of the decision certitudes of the nodes that satisfies a given near consensus specification is nonconvex. So far, no results have been reported on the near consensus complex social networks.

The rest of this paper is organized as follows. A model of complex social networks and eigen theories are reviewed in Section II and Section III, respectively. In Section IV, a near consensus complex social network is defined. By using eigen theories, the relationships between the vectors representing the steady state values of the decision certitudes of the nodes, the influence weight matrix and the set of vectors representing the initial state values of the decision certitudes of the nodes that satisfies a given near consensus specification are characterized. Finally, a conclusion is drawn in Section V.

\section{REVIEW ON A MODEL OF COMPLEX SOCIAL NETWORKS}

Let $N$ be the total number of nodes of a complex social network. Denote $x_{n}(k)$ as the decision certitude of the $n^{\text {th }}$ node of the complex social network at the time index $k$, where $n=0,1, \cdots, N-1$ and $k \geq 0$. Here, when " $x_{n}(k)$ is close to -1 ", the decision of the $n^{\text {th }}$ node at the time index $k$ tends to vote against a given proposal, while when " $x_{n}(k)$ is close to 1 ", the decision of the $n^{\text {th }}$ node at the time index $k$ tends to vote for the given proposal. Let $w_{m, n}$ for $m=0,1, \cdots, N-1$ and for $n=0,1, \cdots, N-1$ be the influence weight factor between the node $m$ and the node $n$. Then, we have

This paper is supported by an Australia research grant from Australia Research Council. 
$x_{n}(k+1)=\sum_{m=0}^{N-1} w_{n, m} x_{m}(k)$ for $n=0,1, \cdots, N-1$ and for $k \geq 0$. Denote $\mathbf{x}(k) \equiv\left[\begin{array}{lll}x_{0}(k) & \cdots & x_{N-1}(k)\end{array}\right]^{T}$ for $k \geq 0$ as the vector representing the decision certitudes of the nodes at the time index $k$ and $\mathbf{W} \equiv\left[\begin{array}{ccc}w_{0,0} & \cdots & w_{0, N-1} \\ \vdots & \ddots & \vdots \\ w_{N-1,0} & \cdots & w_{N-1, N-1}\end{array}\right]$ as the influence weight matrix, where the superscript ${ }^{T}$ denotes the transpose operator. Then, the dynamics of the complex social network can be written in a matrix form as $\mathbf{x}(k+1)=\mathbf{W} \mathbf{x}(k)$ for $k \geq 0$.

Definition 1. A complex social network is said to achieve an exact consensus property if there exists a $k_{0} \geq 0$ such that $x_{0}(k)=\cdots=x_{N-1}(k)=1$ or $x_{0}(k)=\cdots=x_{N-1}(k)=-1$ for $k \geq k_{0}$.

If a complex social network achieves the exact consensus property, then the decision certitudes of the nodes are all equal and all the nodes have 100\% decision certitudes. However, in practical situations it is difficult to get all the nodes having the same decision certitudes. Even though all the nodes have the same decision certitudes, it is difficult to get the $100 \%$ decision certitudes.

\section{REVIEW ON EIGEN THEORIES FOR EXACT CONSENSUS COMPLEX SOCIAL NETWORKS}

From a complex social network viewpoint, the decision certitudes of the nodes have to converge. Also, the steady state values of the decision certitudes of the nodes could not be all equal to zero. Otherwise, a final conclusion on a given proposal cannot be drawn. If the complex social networks achieve the exact consensus property, then the steady state values of the decision certitudes of the nodes have to be all equal and the steady state values are either 1 or -1 . Define $\mathbf{l} \equiv\left[\begin{array}{lll}1 & \cdots & 1\end{array}\right]^{T}$. In order words, the vector representing the decision certitudes of the nodes have to converge to either $\mathbf{t}$ or $-\mathbf{l}$. However, in general the model of complex social networks discussed in Section II does not guarantee the satisfaction of this property. It is important to characterize the conditions on the influence weight matrix and the set of vectors representing the initial state values of the decision certitudes of the nodes so that the above property is satisfied. To address this problem, eigen theories are applied.

Lemma 1. Let $\xi_{n}$ be the eigenvectors of $\mathbf{W}$ corresponding to the eigenvalues $\lambda_{n}$ for $n=0,1, \cdots, N-1$. Then, $\mathbf{x}(k)$ converges to a nonzero fixed vector for each $\mathbf{x}(0) \in \mathfrak{R}^{N} \backslash\{\boldsymbol{0}\}$, where $\mathfrak{R}^{N}$ denotes the $N$-dimensional Euclidean space, if and only if there is at least one eigenvalue equal to 1 and all other eigenvalues are always having modulus less than 1 .

Lemma 1 states that the necessary and sufficient conditions for the vectors representing the decision certitudes of the nodes to converge to a nonzero fixed vector for each nonzero initial condition are that there is at least one eigenvalue equal to 1 and all other eigenvalues are always having modulus less than 1.
Lemma 2. Suppose that $\mathbf{x}(k)$ converges to a nonzero fixed vector for each $\mathbf{x}(0) \in \mathfrak{R}^{N} \backslash\{\boldsymbol{0}\}$. Denote $\mathbf{x}^{*} \equiv \lim _{k \rightarrow+\infty} \mathbf{x}(k)$. Denote $\mathbf{D} \equiv \operatorname{diag}\left(\lambda_{0}, \cdots, \lambda_{N-1}\right)$ and $\mathbf{T} \equiv\left[\begin{array}{lll}\boldsymbol{\xi}_{0} & \cdots & \boldsymbol{\xi}_{N-1}\end{array}\right]$. Assume that $\mathbf{W}$ is diagonalizable, that is $\mathbf{W}=\mathbf{T D T}^{-1}$. Let the first $r$ columns of $\mathbf{T}$ be the first $r$ eigenvectors of $\mathbf{W}$ corresponding to the eigenvalues equal to 1 . Define $\mathbf{V} \equiv\left[\begin{array}{lll}\xi_{0} & \cdots & \xi_{r-1}\end{array}\right]$, $\mathbf{V}^{\prime} \equiv\left[\begin{array}{lll}\xi_{r} & \cdots & \boldsymbol{\xi}_{N-1}\end{array}\right]$ and $\widetilde{\mathbf{a}} \equiv\left[\begin{array}{c}\mathbf{a} \\ \mathbf{a}^{\prime}\end{array}\right] \equiv \mathbf{T}^{-1} \mathbf{x}(0)$, where $\mathbf{a} \in C^{r}$ and $\mathbf{a}^{\prime} \in C^{N-r}$. Here, $C^{r}$ denotes the set of all $r$-dimensional complex-valued vectors. Then, $\mathbf{x}^{*}=\mathbf{V a}$. Furthermore, $\lim _{k \rightarrow+\infty} \mathbf{W}^{k} \mathbf{x}(0)=\mathbf{x}^{*}$ for each $\mathbf{x}(0) \in\left\{\mathbf{x}^{*}+\mathbf{V}^{\prime} \mathbf{a}^{\prime \prime}\right.$, where $\left.\mathbf{a}^{\prime \prime} \in C^{N-r}\right\}$.

Lemma 2 reveals that, for a given $\mathbf{x}(0) \neq \mathbf{0}$ and a diagonalizable $\mathbf{W}$, if $\mathbf{x}(k)$ converges to $\mathbf{x}^{*} \neq \mathbf{0}$, then $\mathbf{x}^{*}$ is uniquely defined by $\mathbf{x}(0)$ and $\mathbf{W}$, in which the relationship is governed by $\mathbf{x}^{*}=\mathbf{V a}=\mathbf{V}\left[\operatorname{diag}\left(\left[\begin{array}{lll}1 & \cdots & 1\end{array}\right]\right) \quad \mathbf{0}\right] \mathbf{T}^{-1} \mathbf{x}(0)$. In other words, the vector representing the steady state values of the decision certitudes of the nodes can only be expressed as a linear combination of the eigenvectors of the influence matrix corresponding to the eigenvalues equal to one. As $\left[\begin{array}{c}\mathbf{a} \\ \mathbf{a}^{\prime}\end{array}\right]=\mathbf{T}^{-1} \mathbf{x}(0)$, the vector consisting of the linear combination coefficients (the vector a) is clearly dependent on the initial condition. If a complex social network achieves the exact consensus property, then $\mathbf{x}^{*}=\mathbf{V}\left[\operatorname{diag}\left(\left[\begin{array}{lll}1 & \cdots & 1\end{array}\right]\right) \quad \mathbf{0}\right] \mathbf{T}^{-1} \mathbf{x}(0)=\mathbf{\imath}$ or $\mathbf{x}^{*}=\mathbf{V}\left[\operatorname{diag}\left(\left[\begin{array}{lll}1 & \cdots & 1\end{array}\right]\right) \mathbf{0}\right] \mathbf{T}^{-1} \mathbf{x}(0)=-\mathbf{l}$. In other words, if a complex social network achieves the exact consensus property, then $\mathbf{\imath}$ or $-\mathbf{l}$ can only be expressed as a linear combination of the eigenvectors of the influence matrix corresponding to the eigenvalues equal to one and the vector consisting of the linear combination coefficients is dependent on the initial condition. On the other hand, for a given $\mathbf{x}^{*} \neq \mathbf{0}$ and a diagonalizable $\mathbf{W}, \mathbf{x}(0)$ is not uniquely defined. Let $S_{\left\{\xi_{r}, \cdots, \xi_{N-1}\right\}}$ be the subspace spanned by the set of vectors $\left\{\xi_{r}, \cdots, \xi_{N-1}\right\}$, and let $S_{\left\{\xi_{r}, \cdots, \xi_{N-1}\right\}}^{\mathbf{x}^{*}}$ be the linear translation of $S_{\left\{\xi_{r}, \cdots, \xi_{N-1}\right\}}$ by the vector $\mathbf{x}^{*} \cdot S_{\left\{\xi_{r}, \cdots, \xi_{N-1}\right\}}^{\mathbf{x}^{*}}$ is called a linear variety of $S_{\left\{\xi_{r}, \cdots, \xi_{N-1}\right\}}$. Obviously, $S_{\left\{\xi_{r}, \cdots, \xi_{N-1}\right\}}^{\mathbf{x}^{*}}=\left\{\mathbf{x}^{*}+\mathbf{V}^{\prime} \mathbf{a}^{\prime \prime}\right.$, where

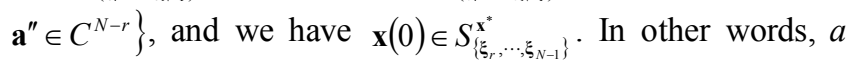
condition for the vector representing the decision certitudes of the nodes to converge to a given vector $\mathbf{x}^{*}$ is that the vector representing the initial state values of the decision certitudes of the nodes have to be in the linear translated subspace spanned by the set of eigenvectors of the influence matrix corresponding to the eigenvalues NOT equal to one and the linear translation is exactly represented by the vector $\mathbf{x}^{*}$. If the complex social networks achieve the exact consensus property, then $\mathbf{x}(0) \in S_{\left\{\xi_{r}, \cdots, \xi_{N-1}\right\}}^{\mathbf{1}} \cup S_{\left\{\xi_{r}, \cdots, \xi_{N-1}\right\}}^{-1}$. In other words, if a complex social network achieves the exact consensus property, then the vector representing the initial state values of the decision certitudes of the nodes have to be in the union of two linear translated subspaces spanned by the set of 
eigenvectors of the influence matrix corresponding to the eigenvalues NOT equal to one and the linear translations are exactly represented by the vector $\mathbf{l}$ and $-\mathbf{l}$.

For the completeness, it is worth noting that exist works on complex social networks usually assume the following condition: $\sum_{n=0}^{N-1} w_{m, n}=1$ for each $m \in\{0,1, \cdots, N-1\}$. A necessary condition between the eigenvectors of $\mathbf{W}$ and the above condition is summarized in the following lemma.

Lemma 3. Suppose that $\mathbf{W}$ is diagonalizable. Assume that there exists a $j \in\{0, \cdots, N-1\}$ such that $\xi_{j}= \pm \mathbf{l}$ corresponding to $\lambda_{j}=1$. Then, $\sum_{n=0}^{N-1} w_{m, n}=1$ for each $m \in\{0,1, \cdots, N-1\}$.

It is worth noting that this condition is not a necessary and sufficient condition for complex social networks to achieve the exact consensus property. Even though Lemma 3 is satisfied, the decision certitudes of the nodes could diverge. In this case, the complex social networks do not achieve the exact consensus property.

\section{Near Consensus COMPlex Social Networks}

Now, the exact consensus property of complex social networks is extended to a near consensus property.

Definition 2. Let $x_{i}^{*}$ be the $(i+1)^{\text {st }}$ element of $\mathbf{x}^{*}$, where $i=0, \cdots, N-1$. For a given threshold value of $0 \leq \delta \leq 1$, if there exist $k_{1}, 0 \leq k_{1} \leq N$, different values of $i \in\{0, \cdots, N-1\}$ such that $1 \geq x_{i}^{*} \geq 1-\delta$, or alternatively, if there exist $k_{1}$, $0 \leq k_{1} \leq N$, different values of $i \in\{0, \cdots, N-1\}$ such that $\delta-1 \geq x_{i}^{*} \geq-1$, then the complex social network is said to achieve a $k_{1} / N$ near consensus property with the threshold value of $1-\delta$. Let $X_{\delta, k_{1}}$ be the set consisting of all such $\mathbf{x}^{*}$.

$k_{1} / N$ is the percentage of the total number of nodes that the steady state values of the decision certitudes of these nodes are either between $1-\delta$ and 1 or between -1 and $\delta-1$. Different complex social networks have different minimum percentage of the total number of nodes to pass or to deny a given proposal. The value of $k_{1}$ should be set accordingly. Also, different complex social networks require different threshold values on the steady state values of the decision certitudes of the nodes to vote for or against the given proposal. The threshold value of $\delta$ should be set accordingly. For example, if a complex social network requires at least $70 \%$ of the total number of nodes to pass or to deny a given proposal and each node is required having at least over $90 \%$ of the steady state value of its decision certitude to vote for or against the given proposal, then $k_{1} / N=0.7$ and $\delta=0.1$. Obviously, if $\delta=0$ and $k_{1}=N$, then the near consensus property reduces to the exact consensus property. Note that $X_{\delta, k_{1}}$ is, in general, nonconvex.

As most complex social networks are scale free networks, we consider scale free complex social networks in this paper.
Lemma 4. Suppose that there are $n$ hubs with high connectivity. Assume, without loss of generality, that these $n$ hubs are located at the first $n$ nodes of the complex social network. Also, assume that the decisions of these $n$ hubs are influenced by each other only, but not by the other nodes (i.e., $w_{i, m}=0$ for each $i \in\{0,1, \cdots, n-1\}$ and for each $m \in\{n, n+1, \cdots, N-1\})$. Moreover, the decisions of the following $k$ nodes are influenced by these $n$ hubs and these $k$ nodes themselves, but not by the other nodes. Obviously, there exist $\quad \mathbf{W}_{\mathbf{A}} \in \mathfrak{R}^{n \times n}, \quad \mathbf{W}_{\mathbf{B}} \in \mathfrak{R}^{k \times k}, \quad \mathbf{W}_{\mathbf{C}} \in \mathfrak{R}^{(N-n-k) \times(N-n-k)}$, $\mathbf{W}_{\mathbf{D}} \in \mathfrak{R}^{k \times n}, \quad \mathbf{W}_{\mathbf{E}} \in \mathfrak{R}^{(N-n-k) \times n}$ and $\quad \mathbf{W}_{\mathbf{F}} \in \mathfrak{R}^{(N-n-k) \times k}$ such that $\mathbf{W} \equiv\left[\begin{array}{ccc}\mathbf{W}_{\mathbf{A}} & \mathbf{0} & \mathbf{0} \\ \mathbf{W}_{\mathbf{D}} & \mathbf{W}_{\mathbf{B}} & \mathbf{0} \\ \mathbf{W}_{\mathbf{E}} & \mathbf{W}_{\mathbf{F}} & \mathbf{W}_{\mathbf{C}}\end{array}\right]$. Assume that $\mathbf{W}_{\mathbf{A}}, \mathbf{W}_{\mathbf{B}}$ and $\mathbf{W}_{\mathbf{C}}$ are diagonalizable. Let $\xi_{\mathbf{A}_{i}}$ be the eigenvectors of $\mathbf{W}_{\mathbf{A}}$ corresponding to the eigenvalues $\lambda_{\mathbf{A}_{i}}$ for $i=0,1, \cdots, n-1$. Let $\xi_{\mathbf{B}_{i}}$ be the eigenvectors of $\mathbf{W}_{\mathbf{B}}$ corresponding to the eigenvalues $\lambda_{\mathbf{B}_{i}}$ for $i=0,1, \cdots, k-1$, and let $\xi_{\mathbf{C}_{i}}$ be the eigenvectors of $\mathbf{W}_{\mathbf{C}}$ corresponding to the eigenvalues $\lambda_{\mathbf{C}_{i}}$ for

$$
\begin{aligned}
& i=0,1, \cdots, N-n-k-1 \text {. Define } \mathbf{T}_{\mathbf{C}} \equiv\left[\begin{array}{ccc}
\mathbf{0} & \cdots & \mathbf{0} \\
\mathbf{0} & \cdots & \mathbf{0} \\
\boldsymbol{\xi}_{\mathbf{C}_{0}} & \cdots & \xi_{\mathbf{C}_{(N-n-k-1)}}
\end{array}\right] \text {, } \\
& \mathbf{T}_{\mathbf{B}} \equiv\left[\begin{array}{ccc}
\mathbf{0} & \cdots & \mathbf{0} \\
\boldsymbol{\xi}_{\mathbf{B}_{0}} & \cdots & \boldsymbol{\xi}_{\mathbf{B}_{k-1}} \\
\left(\lambda_{\mathbf{B}_{0}} \mathbf{I}-\mathbf{W}_{\mathbf{C}}\right)^{-1} \mathbf{W}_{\mathbf{F}} \xi_{\mathbf{B}_{0}} & \cdots & \left(\lambda_{\mathbf{B}_{k-1}} \mathbf{I}-\mathbf{W}_{\mathbf{C}}\right)^{-1} \mathbf{W}_{\mathbf{F}} \boldsymbol{\xi}_{\mathbf{B}_{k-1}}
\end{array}\right],
\end{aligned}
$$

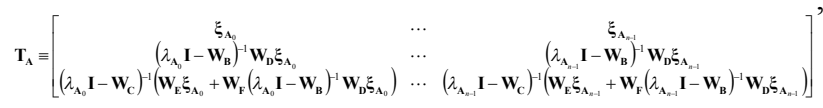

$$
\begin{aligned}
& \mathbf{T}^{\prime} \equiv\left[\begin{array}{lll}
\mathbf{T}_{\mathbf{C}} & \mathbf{T}_{\mathbf{B}} & \mathbf{T}_{\mathbf{A}}
\end{array}\right] \text { and } \mathbf{D}^{\prime} \equiv \operatorname{diag}\left(\lambda_{\mathbf{C}_{0}}, \cdots, \lambda_{\mathbf{C}_{N-n-k}}, \lambda_{\mathbf{B}_{0}}, \cdots, \lambda_{\mathbf{B}_{k-1}}, \lambda_{\mathbf{A}_{0}}, \cdots, \lambda_{\mathbf{A}_{n-1}}\right) \text {. }
\end{aligned}
$$
Then, $\mathbf{W}$ is diagonalizable given by $\mathbf{W}=\mathbf{T}^{\prime} \mathbf{D}^{\prime} \mathbf{T}^{-1}$.

From Lemma 4, we see that the eigenvectors and the corresponding eigenvalues of $\mathbf{W}$ can be found efficiently through finding the eigenvectors and the corresponding eigenvalues of $\mathbf{W}_{\mathbf{A}}, \mathbf{W}_{\mathbf{B}}$ and $\mathbf{W}_{\mathbf{C}}$. This is important, because the dimensions of $\mathbf{W}_{\mathbf{A}}, \mathbf{W}_{\mathbf{B}}$ and $\mathbf{W}_{\mathbf{C}}$ are much lower than that of $\mathbf{W}$, and scale free networks usually consist of many nodes.

The set of eigenvalues of $\mathbf{W}$ is the union of the sets of eigenvalues of $\mathbf{W}_{\mathbf{A}}, \mathbf{W}_{\mathbf{B}}$ and $\mathbf{W}_{\mathbf{C}}$ only. According to Lemma $1, \mathbf{x}(k)$ converges to a nonzero fixed vector for each $\mathbf{x}(0) \in \mathfrak{R}^{N} \backslash\{\boldsymbol{0}\}$ if and only if there is at least one eigenvalue in the set $\left\{\lambda_{\mathbf{C}_{0}}, \cdots, \lambda_{\mathbf{C}_{N-n-k}}, \lambda_{\mathbf{B}_{0}}, \cdots, \lambda_{\mathbf{B}_{k-1}}, \lambda_{\mathbf{A}_{0}}, \cdots, \lambda_{\mathbf{A}_{n-1}}\right\}$ equal to 1 and all other eigenvalues are always having modulus less than 1 .

By combining the results in Lemma 2 and Lemma 4, we obtain the relationships between the vectors representing the steady state values of the decision certitudes of the nodes and the influence weight matrix of a near consensus complex 
social network. Re-arrange the columns in $\mathbf{T}^{\prime}$ and the corresponding diagonal elements in $\mathbf{D}^{\prime}$ to give, respectively, a new matrix $\mathbf{T}$ and a new matrix $\mathbf{D}$ such that the first $r$ columns of $\mathbf{T}$ are the first $r$ eigenvectors of $\mathbf{W}$ corresponding to the eigenvalues which are equal to 1 . Then, the vectors representing the steady state values of the decision certitudes of the nodes are a linear combination of the first $r$ eigenvectors of $\mathbf{W}$ corresponding to the eigenvalues equal to one. The corresponding linear combination coefficients are dependent on the initial condition.

Corollary 1. If the steady state values of the decision certitudes of some of these $n$ hubs are nonzero, then there exist an $i \in\{0,1, \cdots, n-1\}$ such that $\lambda_{\mathbf{A}_{i}}=1$.

Corollary 1 indicates that if the steady state values of the decision certitudes of some of these $n$ hubs are nonzero, then at least one eigenvalue of the influence matrix is equal to one. In order to check whether the steady state values of the decision certitudes of some of these $n$ hubs are nonzero, it is more easy to check whether there exist eigenvalues of $\mathbf{W}_{\mathbf{A}}$ which are equal to one.

Theorem 1. The set of vectors representing the initial state values of the decision certitudes of the nodes that satisfies a given near consensus specification is:

$F_{\delta, k_{1}} \equiv\left\{\mathbf{V}\left(\mathbf{V}^{T} \mathbf{V}\right)^{-1} \mathbf{V}^{T} \mathbf{x}^{*}+\mathbf{V}^{\prime} \mathbf{a}^{\prime \prime}\right.$, where $\mathbf{x}^{*} \in X_{\delta, k_{1}}$ and $\left.\mathbf{a}^{\prime \prime} \in C^{N-r}\right\}$.

Based on Definition 2, $X_{\delta, k_{1}}$ is uniquely defined for a given near consensus specification on $\delta$ and $k_{1}$. However, not all the vectors in $X_{\delta, k_{1}}$ can be expressed as a linear combination of the eigenvectors of $\mathbf{W}$ corresponding to the eigenvalues equal to one. Theorem 1 characterizes the set of vectors representing the initial state values of the decision certitudes of the nodes that satisfies a given near consensus specification.

Lemma 5. Consider a special case where $n=2$ and $\mathbf{W}_{\mathbf{B}}$ is a diagonal matrix. Assume that $\mathbf{x}(k)$ converges to a nonzero fixed vector for each $\mathbf{x}(0) \in \mathfrak{R}^{N} \backslash\{\mathbf{0}\}$. Also, assume that $\sum_{n=0}^{N-1} w_{m, n}=1$ for each $m \in\{0,1, \cdots, N-1\}$. Let $k_{1} \equiv k+2$. Then, $x_{0}^{*}=x_{1}^{*}=\cdots=x_{k_{1}-1}^{*}$. Define $\quad x_{0}^{*}=x_{1}^{*}=\cdots=x_{k_{1}-1}^{*} \equiv c \quad$ and $\delta \equiv 1-|c|$. Suppose that $1 \geq|c| \geq 0$. Then, the complex social network achieves at least the $k_{1} / N$ near consensus property with the threshold value larger than or equal to $1-\delta$.

Lemma 5 refers to the case where there are two hubs with high connectivity, in which the decisions of these two hubs are influenced by one another, but not by the other nodes. Also, the decisions of the following $k$ nodes are influenced by these two hubs, but not by themselves and by the other nodes. Moreover, the sums of the influence weight factors of all the nodes are normalized to one and the vectors representing the decision certitudes of the nodes converge to a nonzero vector. Lemma 5 reveals that $X_{\delta, k_{1}}$ consists of two hyperplanes (i.e.,
$X_{\delta, k_{1}}=\left\{\begin{array}{llllll}c & \cdots & c & x_{k}^{*} & \cdots & x_{N-1}^{*}\end{array}\right]^{T}: 1 \geq|c| \geq 0$ or $\left.\left.0 \geq-|c| \geq-1\right\}\right)$.

Note that no conclusion can be drawn on the decisions at the rest of $N-k_{1}$ nodes. Fig. 1 shows a numerical computer simulation result on the steady state values of the decision certitudes of the last ten nodes of the scale free network. It can be seen from Fig. 1 that the steady state values of the decision certitudes of some of these ten nodes are not equal, which show that the exact consensus property is not satisfied. On the other hand, those nodes which are influenced by these two hubs only are all equal and the value is equal to that of these two hubs. This implies that the near consensus property is satisfied.

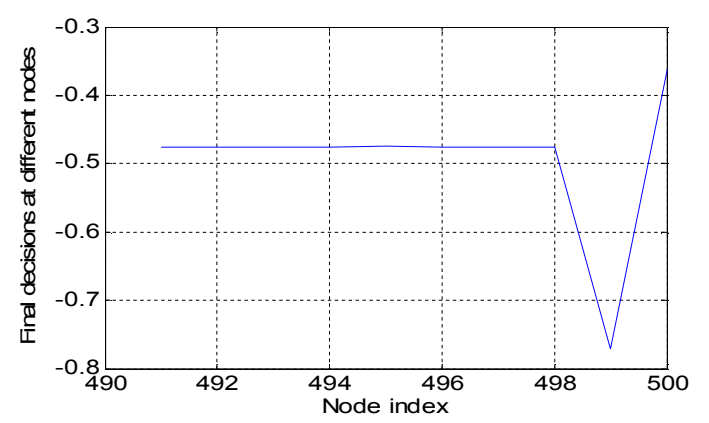

Fig. 1. Steady state values of the decision certitudes of the last ten nodes. The steady state values of the decision certitudes of the other nodes have not been shown on the figure because they have consensus with the hubs.

\section{CONCLUSION}

In this paper, an exact consensus property of complex social networks is extended to a near consensus property, which is more realistic and practical. It is shown that the vectors representing the steady state values of the decision certitudes of the nodes are linear combinations of the eigenvectors of the influence weight matrix corresponding to the eigenvalues equal to 1 . Furthermore, the set of vectors representing the initial state values of the decision certitudes of the nodes that satisfies a given near consensus specification is characterized.

\section{REFERENCES}

[1] J. Yu, E. Noel and K. W. Tang, "A graph theoretic approach to ultrafast information distribution: Borel Cayley graph resizing algorithm," in press in Computer Communications, 2010.

[2] J. Zhou and Q. Wang, "Convergence speed in distributed consensus over dynamically switching networks," Automatica, vol. 45, pp. 14551461, 2009.

[3] R. J. Johnston, C. J. Pattie, D. F. L. Dorling, I. MacAllister, H. Tunstall and D. J. Rossiter, "Social locations, spatial locations and voting at the 1997 British general election: evaluating the sources of Conservative support," Political Geography, vol. 20, pp. 85-111, 2001.

[4] Wenwu Yu, Guanrong Chen, Ming Cao and Jürgen Kurths, "Secondorder consensus for multiagent systems with directed topologies and nonlinear dynamics," IEEE Transactions on Systems, Man, and Cybernetics-Part B: Cybernetics, vol. 40, no. 3, pp. 881-891, 2010.

[5] Wenwu Yu, Guanrong Chen and Ming Cao, "Some necessary and sufficient conditions for second-order consensus in multi-agent dynamical systems," Automatica, vol. 46, pp. 1089-1095, 2010. 
